\title{
Is the Airtraq optical laryngoscope effective in tracheal intubation by novice personnel?
}

\author{
Sang-Jin Park, Won Ki Lee, and Deok Hee Lee \\ Department of Anesthesiology and Pain Medicine, College of Medicine, Yeungnam University, Daegu, Korea
}

Background: Macintosh laryngoscopic intubation is a lifesaving procedure, but a difficult skill to learn. The Airtraq optical laryngoscope (AOL) is a novel intubation device with advantages over the direct laryngoscope for untrained personnel in a manikin study. We compared the effectiveness of AOL with Macintosh laryngoscope for tracheal intubation by novice personnel.

Methods: We selected 37 medical students with no prior tracheal intubation experience and educated them on using both laryngoscopes. Seventy-four patients were randomly divided into two groups (group A: AOL, group M: Macintosh laryngoscope). We recorded the tracheal intubation success rate, intubation time, number of attempts, intubation difficulty scale, and adverse effects.

Results: The total success rate was similar in the two groups, but the success rate at first attempt was higher in group A $(\mathrm{P}<0.01)$. Group A also showed reduced duration and attempts at intubation, as well as adverse effects such as oral cavity injury. Additionally, participant reports indicated that using the AOL was easier than the Macintosh laryngoscope $(\mathrm{P}<0.01)$.

Conclusions: The AOL is a more effective instrument for tracheal intubation than Macintosh laryngoscope when used by novice personnel. (Korean J Anesthesiol 2010; 59: 17-21)

Key Words: Airtraq optical laryngoscope, Intubation, Macintosh laryngoscope.

Received: March 24, 2010. Revised: April 14, 2010. Accepted: April 16, 2010.

Corresponding author: Deok Hee Lee, M.D., Department of Anesthesiology and Pain Medicine, College of Medicine, Yeungnam University, Daemyeong 5-dong, Nam-gu, Daegu 705-717, Korea. Tel: 82-53-620-3368, Fax: 82-53-626-5275, E-mail: dhlee415@ynu.ac.kr The 86th annual scientific meeting of the Korean Society of Anesthesiologists, 2009. International convention center Jeju (ICC Jeju). This research was supported by the Yeungnam University research grants in 2009.

() This is an open-access article distributed under the terms of the Creative Commons Attribution Non-Commercial License (http:// creativecommons.org/licenses/by-nc/3.0/), which permits unrestricted non-commercial use, distribution, and reproduction in any medium, provided the original work is properly cited. 


\section{Introduction}

Endotracheal intubation is performed using the laryngoscope at a sniffing position to secure a close proximity view of the glottis and align the oral, pharyngeal, and laryngeal axes. Tracheal intubation requires a great deal of clinical experience to master, such that performing intubation more than 47 times only produces a $90 \%$ success rate of intubation [1]. Consequently, other instruments that facilitate endotracheal intubation may replace the conventional direct laryngoscope, including the Airtraq optical laryngoscope (AOL, Airtraq ${ }^{\circledR}$, Guangzhou Intmed Medical Appliance Co., Guangzhou, China) (Fig. 1). The AOL is similar to the direct laryngoscope, but consists of a body with built-in lens and mirror, a view-finder, and a rail for fixing the tracheal tube. The attached lens allows direct observation of the larynx and facilitates endotracheal intubation. In a study using a training dummy, the success rate of AOL intubation was significantly higher and the time to secure the airway was also shorter than using the Macintosh direct laryngoscope [2]. However, tracheal intubation in real patients is challenging, especially for novices, even with sufficient training in dummies [1]. Therefore, we compared the performance of the AOL and Macintosh laryngoscopes for endotracheal intubation by novice physicians for general anesthesia.

\section{Materials and Methods}

The present study was approved by our Institutional Bioethics Board of Clinical Research and the study population consisted

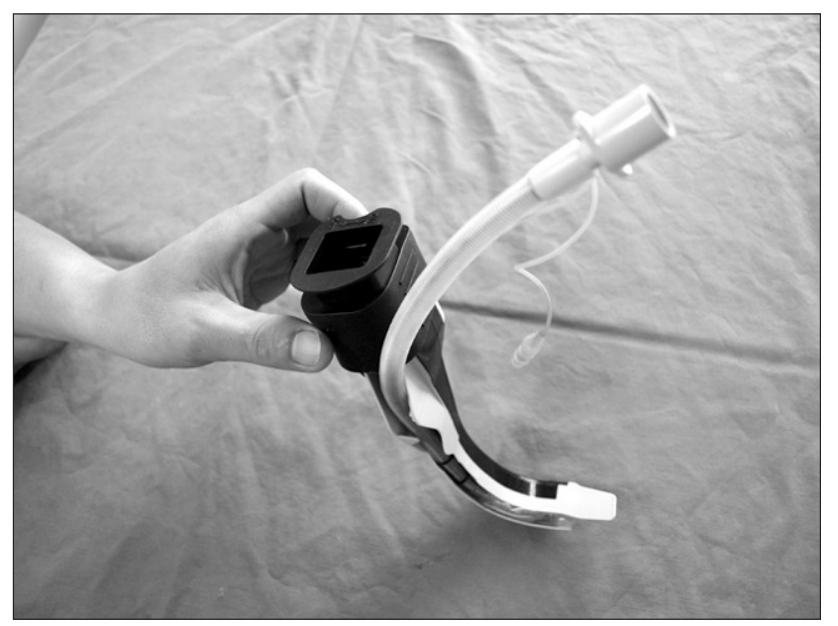

Fig. 1. Photograph of the Airtraq optical laryngoscope (AOL). The blade of the AOL is anatomically shaped and consists of two parallel channels. One channel performs as the placing and inserting of the endotracheal tube. The other channel houses a distal lens and LED light. The image of the glottic structures and the tip of the tracheal tube is transferred to a viewfinder. of 37 medical students on clinical training without endotracheal intubation experience. The students received orientation for endotracheal intubation using both the Macintosh direct laryngoscope and the AOL through audio-visual materials, and then performed 10 endotracheal intubations on intubationtraining manikins $\left(\mathrm{Ambu}^{\circledR}\right.$ intubation trainer, Ambu International A/S, Kopenhagen, Denmark) under the guidance of an anesthesiologist with experience employing the AOL for tracheal intubation 100 or more times. Afterwards, each student performed endotracheal intubation on two real patients with both laryngoscopes and the attending anesthesiologist present. For allocation of students to the patient for intubation, patients were given one of a series of numbers and the participant was asked to choose any two numbers at random. A coin toss was used to decide AOL or Macintosh methods for two groups: Group M with the Macintosh laryngoscope and Group A with the AOL.

The subjects for endotracheal intubation included patients between the ages of 18-60 years, with American Society of Anesthesiologists physical status I or II, who were scheduled to undergo surgery under general anesthesia. Prior to their enrollment, informed written (parental) consent following full explanation of the purpose and the procedure of this study was obtained. Those patients at preoperative examination were excluded who had cardiopulmonary disease such as hypertension, angina pectoris, or asthma, who had potential risk of pulmonary aspiration in tracheal intubation due to pregnancy or ascites, who had no teeth or loose teeth, or who were expected to have difficulty with endotracheal intubation. Also excluded were those patients who were assessed as grade 3 or over on the classification by Cormack \& Lehane when, after induction of anesthesia, an anesthesiologist examined the exposure view of the glottis and the epiglottis with the Macintosh direct laryngoscope.

On arrival into the operating room, electrocardiogram, noninvasive blood pressure analysis, and pulse oximetry were attached. After propofol 1.5-2 mg/kg and rocuronium $1 \mathrm{mg} /$ $\mathrm{kg}$ were injected intravenously for induction of anesthesia, mask ventilation was performed with $100 \%$ oxygen and $3-4$ vol\% sevoflurane for about 2 minutes and 30 seconds. Then, the anesthesiologist, who supervised endotracheal intubation training for the participant students, evaluated the exposure degree of the glottis and the epiglottis of the patients with using the Macintosh direct laryngoscope to record the grade of Cormack \& Lehane classification, either grade 1 or 2 , followed by another minute of mask ventilation. Afterwards, each participant began to perform endotracheal intubation using the Macintosh direct laryngoscope or the AOL. No assistance was provided during the intubation. Endotracheal tubes of inner diameter (ID) size $7.5 \mathrm{~mm}$ were used for male adult patients, 
while those of ID $7.0 \mathrm{~mm}$ for female patients.

For each participant, the number of tracheal intubation attempts and the duration were recorded. Such cases were considered failure as when saturation measured by pulse oximeter $\left(\mathrm{SpO}_{2}\right)$ decreased to $90 \%$ or below at intubation, when intubation was not completed within 1 minute and 30 seconds, when incorrect intubation was made into the esophagus, followed by another attempt 1 minute later, and when three attempts at intubation on one patient did not succeed. We recorded time to successful intubation from opening the mouth to the first appearance of normal wave capnography. Mask ventilation periods were excluded from the total intubation time. We recorded the number of esophageal intubations and episodes such as hypoxia $\left(\mathrm{SaO}_{2} \leq 90 \%\right)$, damage to the teeth, intraoral injury (determined by the presence of blood detected on the oral suction and laryngoscope after intubation), pulmonary aspiration, or the development of spasm in the bronchus and the larynx. In addition, participants rated the ease of use of each device on a visual analogue scale (VAS; from 0 , the easiest imaginable to $100 \mathrm{~mm}$, the most extreme difficulty imaginable).

Statistical analysis was performed using SPSS ver. 17.0 (SPSS

Table 1. Patient Characteristics

\begin{tabular}{lcc}
\hline & Group A (n=37) & Group M (n=37) \\
\hline Age (yr) & $39.5 \pm 12.3$ & $38.3 \pm 11.9$ \\
Sex $(\mathrm{M} / \mathrm{F})$ & $15 / 22$ & $15 / 22$ \\
Height $(\mathrm{cm})$ & $163.4 \pm 8.6$ & $165.4 \pm 8.6$ \\
Weight $(\mathrm{kg})$ & $62.4 \pm 12.3$ & $64.1 \pm 10.3$ \\
Cormark-Lehanegrade $(1 / 2)$ & $28 / 9$ & $30 / 7$ \\
\hline
\end{tabular}

Values are mean \pm SD or number. Group A: intubated using Airtraq optical laryngoscope, Group M: intubated using Macintosh laryngoscope.

Table 2. Comparison of Tracheal Intubation Using the Airtraq Optical Laryngoscope or the Macintosh Laryngoscope

\begin{tabular}{lcc}
\hline & Group A (n=37) & Group M (n=37) \\
\hline Overall success rate & $35 / 37(94.6)$ & $30 / 37(81.1)$ \\
Intubation time of overall & $58.1 \pm 23.1^{*}$ & $90.3 \pm 39.8$ \\
successful intubation (s) & & \\
Numberof intubation attempts & & \\
1 & $32 / 37(86.5)^{*}$ & $19 / 37(51.4)$ \\
$2 \leq$ & $5 / 37(13.5)^{*}$ & $18 / 37(48.6)$ \\
Intubation time of success at & $51.9 \pm 11.1^{\dagger}$ & $66.1 \pm 21.8$ \\
1st attempt (s) & & \\
Difficulty rating on VAS (cm) & $3.3 \pm 1.8^{*}$ & $4.9 \pm 1.8$ \\
Esophageal intubation & $2 / 37(5.4)$ & $7 / 37(18.9)$ \\
\hline
\end{tabular}

Values are mean \pm SD or number (\%). Group A: intubated using Airtraq optical laryngoscope, Group M: intubated using Macintosh laryngoscope. *P $<0.01$ compared with Group $\mathrm{M} .{ }^{\dagger} \mathrm{P}<0.05$ compared with Group M.
Inc., Chicago, IL, USA). Continuous variables such as age, height, weight, difficulty, and time taken for endotracheal intubation were compared using an unpaired t-test. Categorical variable such as sex, grades of Cormack \& Lehane classification, the number of successful intubations, the number of participants succeeding in intubation at the first attempt, and complication rates were compared using Chi-square test or Fisher's exact test. $\mathrm{P}<0.05$ was considered statistically significant.

\section{Results}

Both groups showed similar demographic data and Cormack \& Lehane classifications (Table 1). The overall success rate of endotracheal intubation was $81.1 \%$ for Group M and $94.6 \%$ for Group A, but were not significantly different (Table 2). Total intubation time was significantly shorter in Group A (58.1 sec) than Group M $(90.3 \mathrm{sec})(\mathrm{P}<0.01)$. The time taken for a successful first attempt was also shorter in Group A (51.9 sec) than Group M (66.1 sec) $(\mathrm{P}<0.05$, Table 2), and the initial success rate was higher in Group A (86.5\%) than Group M (51.4\%) $(\mathrm{P}<0.01$, Table 2). Group A showed a significantly lower difficulty rating (3.3) than Group M (4.9) on a VAS (P < 0.01 , Table 2). Incorrect tube placement into the esophagus occurred 7 times in Group M and 2 times in Group A, which did not mean much statistically.

For complications, intraoral injury at intubation occurred 11 times in Group M (29.7\%) and 4 times in Group A (10.8\%) (P < 0.05 , Table 3 ), but were not serious enough to influence the vital signs or to cause postoperative discomfort. We did not observe other complications such as pulmonary aspiration, hypoxia $\left(\mathrm{SaO}_{2} \leq 90 \%\right)$, damage to the teeth, or spasm in the bronchus and larynx.

\section{Discussion}

We compared the ease of technical acquisition by medical

Table 3. Comparison of Complication Rates during Tracheal Intubation Using the Airtraq Optical Laryngoscope and the Macintosh Laryngoscope

\begin{tabular}{lcc}
\hline & $\begin{array}{c}\text { Group A } \\
(\mathrm{n}=37)\end{array}$ & $\begin{array}{c}\text { Group M } \\
(\mathrm{n}=37)\end{array}$ \\
\hline Oral cavity injury & $4 / 37(10.8)^{*}$ & $11 / 37(29.7)$ \\
Hypoxia & 0 & 0 \\
Pulmonary aspiration & 0 & 0 \\
Dental injury & 0 & 0 \\
Laryngospasm or bronchospasm & 0 & 0
\end{tabular}

Values are number (\%). Group A: intubated using Airtraq optical laryngoscope, Group M: intubated using Macintosh laryngoscope. *P $<0.05$ compared with Group M. 
school students with no prior intubation experience using the Macintosh direct laryngoscope and the AOL. The AOL had a higher initial success rate, required less time for successful intubation, and fewer intubation complications.

In a comparison of AOL and Macintosh intubation on manikins with a normal airway, the entire success rate of intubation was 97.5\% for both devices, but the success rate at first attempt was 95\% for the AOL Group and $82.5 \%$ for the Macintosh Group, not significantly different [2]. In contrast, we found significantly higher first attempt success rates in Group A (86.5\%) than Group M (51.4\%), although overall success rates were similar (Group A, 94.6\%; Group M, 81.1\%) and considerably lower than in the study of Maharaj et al. [2]. The different success rates we observed probably resulted from the fact that we worked with real patients, as opposed to manikins. Different from our study, Maharaj et al. [2] employed as subjects for intubation the manikins that were used for the training of endotracheal intubation, where participants seemed to have performed endotracheal intubation with ease based on the past experience and success rates seemed to be independent of the device. Furthermore, diversity in patient intubation conditions and the psychological burden of inducing complications may cause difficulty in intubating patients.

Novice laryngoscopists show low (68\%) success rates of endotracheal intubation in patients, even after extensive practice on manikins [3]. According to another study, overall success rates of intubation reached just $86 \%$ when novice laryngoscopists performed 5 attempts using the conventional direct laryngoscope even in normal adult patients [1]. We found an overall success rate with AOL (94.6\%) higher than other reports with the Macintosh laryngoscope [1,3], as well as higher initial success rates $(86.5 \%$ for the AOL group and $51.4 \%$ for the Macintosh group), which indicates that the AOL performs better than the Macintosh direct laryngoscope with respect to enhancing the success rate of intubation for novice operators.

Failure of prompt endotracheal intubation may cause pulmonary aspiration of gastric contents or hypoxemia. Therefore, the duration of intubation is an important performance metric for intubation devices, as is the success rate of intubation. In manikins, the time required for endotracheal intubation in using the AOL $(18.7 \mathrm{sec})$ was shorter than the Macintosh laryngoscope $(40.8 \mathrm{sec})$ for novice personnel [2]. However, there was no difference in real patients between the time required for intubation using direct laryngoscope (12.4 sec) and AOL (12.2 sec) [4]. We observed a longer duration of intubation, but both the total time taken for endotracheal intubation and the time required for intubation achieved at first attempt were lower in the AOL group (58.1 sec, $51.9 \mathrm{sec}$, respectively) than the Macintosh group (90.3 sec, $66.1 \mathrm{sec}$, respectively). Our cohort included novice laryngoscopists (medical school students), whereas Maharaj et al. [4] observed experienced anesthesiologists who could perform prompt endotracheal intubation regardless of the device. Still, endotracheal intubation using the AOL may enable novice medical personnel to perform intubation more quickly.

Minimizing the risk of complications during intubation is as important as performing prompt and correct endotracheal intubation. Up to $24 \%$ of endotracheal intubation attempts by novice laryngoscopists damage the teeth or mucous layer, despite manikin training [1]. We found a $29.7 \%$ rate of intraoral injury for the Macintosh device but a lower rate (10.8\%) for the AOL device. On the other hand, in manikins with difficult intubations, esophageal intubation occurred less frequently with the AOL device (13\%) than the Macintosh device (65\%) [5]. We did not find a significant difference in esophageal intubation between the Macintosh direct laryngoscope group (7 persons) and the AOL group (2 persons), probably due to restricting the patient population to a Grade 2 or below on the Cormack \& Lehane classification. Intubation of higher-grade patients (Grade 3 or 4) with intubation difficulty may lead to higher rates of esophageal intubation.

Unlike the Macintosh laryngoscope, the AOL provides a view of the glottis without aligning the oral, pharyngeal, and laryngeal axes [2]. With an indirect close proximity view through the distal tip lens of the laryngoscope, this new intubation device allows endotracheal intubation to be performed in any position, even when the "sniffing" position is not possible. In addition, the AOL is easier to use, allows for precise endotracheal intubation, and reduces the number of reattempts after esophageal intubation or failed endotracheal intubation, which enhances initial success rates and reduces total intubation time. The AOL also lessens the time for instrumentation, reduces tension on the larynx [2], and lowers the incidence of damage to the teeth or oral cavity. Despite these advantages, the device is expensive (W150,000 apiece) for a single use device, it cannot be applied for temporomandibular joint dysfunction when the mouth is not opened, and it cannot be used for nasal intubation. In addition, because the AOL is made of plastic rather than metal, excessive force may break the blade.

In summary, we compared the effectiveness of the AOL with the Macintosh direct laryngoscope for endotracheal intubation in patients with a Grade 1 or 2 Cormack \& Lehane classification as performed by novice personnel with no prior experience of endotracheal intubation. The AOL device improved the success rate of endotracheal intubation as well as reduced intubation time, perceived intubation difficulty, and complication rates, suggesting it may be a better device for novice laryngoscopists with insufficient experience with endotracheal intubation in real patients. 


\section{References}

1. Mulcaster JT, Mills J, Hung OR, MacQuarrie K, Law JA, Pytka $\mathrm{S}$, et al. Laryngoscopic intubation: learning and performance. Anesthesiology 2003; 98: 23-7.

2. Maharaj CH, Costello JF, Higgins BD, Harte BH, Laffey JG. Learning and performance of tracheal intubation by novice personnel: a comparison of the Airtraq and Macintosh laryngoscope. Anaesthesia 2006; 61: 671-7.

3. Stewart RD, Paris PM, Pelton GH, Garretson D. Effect of varied training techniques on field endotracheal intubation success rates. Ann Emerg Med 1984; 13: 1032-6.

4. Maharaj CH, O'Croinin D, Curley G, Harte BH, Laffey JG. A comparison of tracheal intubation using the airtraq or the macintosh laryngoscope in routine airway management: a randomised, controlled clinical trial. Anaesthesia 2006; 61: 1093-9.

5. Woollard M, Lighton D, Mannion W, Watt J, McCrea C, Johns I, et al. Airtraq vs standard laryngoscopy by student paramedics and experienced prehospital laryngoscopists managing a model of difficult intubation. Anaesthesia 2008; 63: 26-31 